\title{
Performance Driven Reliable Link Design for Network on Chips
}

\author{
Rutuparna Tamhankar \\ Srinivasan Murali \\ Prof. Giovanni De Micheli
}

Stanford University 


\section{Outline}

- Introduction

$\rightarrow$ Objective

- Logic design and implementation

- Alternative approaches

- Transistor level design

- Timing overheads

- Simulation Results

- Conclusion 


\section{Introduction}

- Wire delay contribution to total delay is increasing

- $6-10$ cycles needed to diagonally cross the chip in $50 \mathrm{~nm}$

- Faster clock cycles, scaling voltages and noisy environment make wires unreliable.

- Unpredictability in wire characteristics results in variation in wire delay

- Noise-induced wire delay consumes greater percentage of useful clock cycle.

- Conservative design approaches that consider worst case operating conditions, result in poor performance. 


\section{Objective of work}

- Motivational example

- A 3-bit adversarial switching pattern (101$>010, \quad 010->101)$ increases wire delay by $50 \%$.

- Conservative approach results in larger clock cycle to account for such delay variations.

- Use aggressive design approach

- Designed for normal (ignoring noise) conditions, tolerate errors caused to noisy environment.

- Use higher clock rate than conservative design or increase spacing between adjacent buffers. 


\section{Timing Error-Tolerant System}

- Timing error-tolerant (Terror) system

- Detects and corrects timing errors with minimal impact on latency.

- Terror systems can tolerate delay variations giving large latency savings (upto 35\%) over traditional retransmission scheme.

- Only transient timing errors are corrected, static errors due to logic faults, soft errors etc. are not corrected. 


\section{Buffered link design}

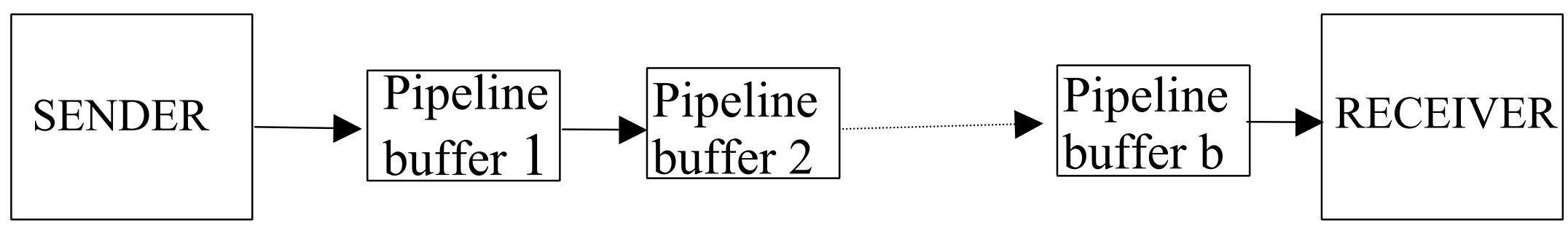

Typical pipelined link design with $\mathrm{b}$ stages

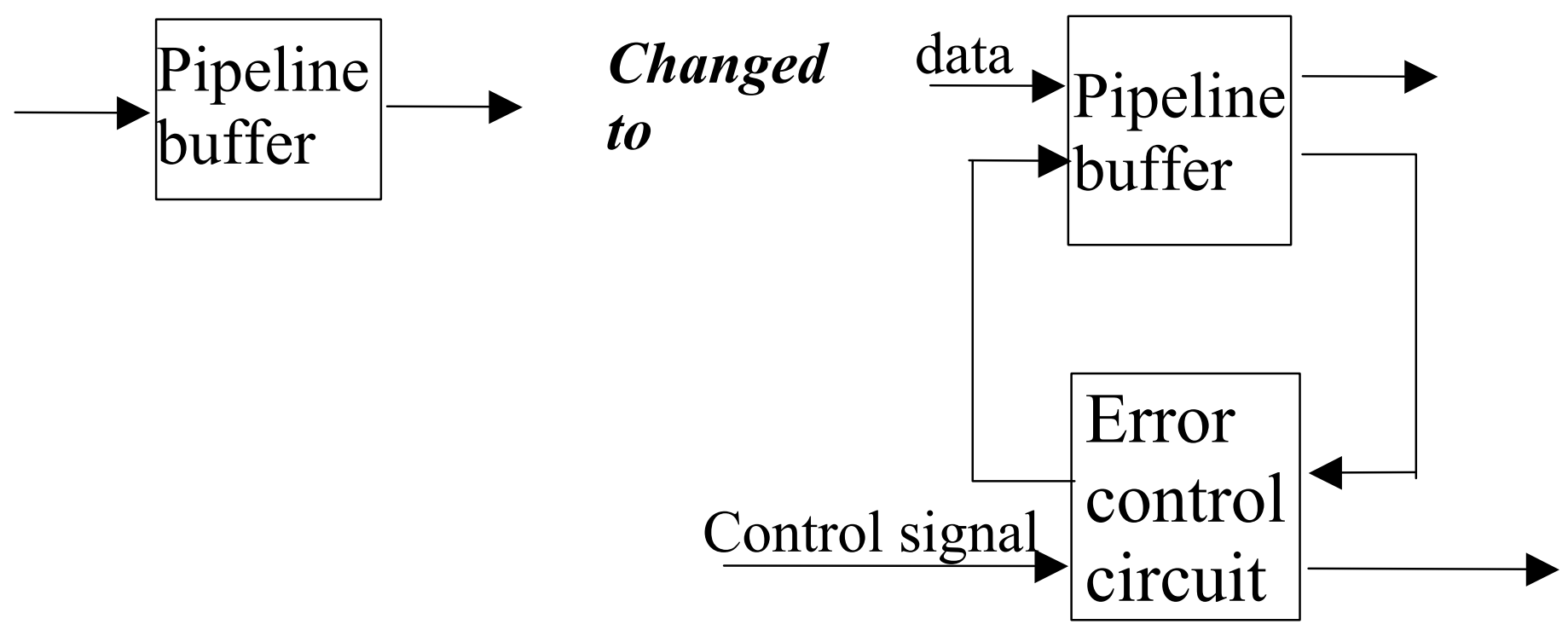

Proposed Design 


\section{Terror Design Principle}

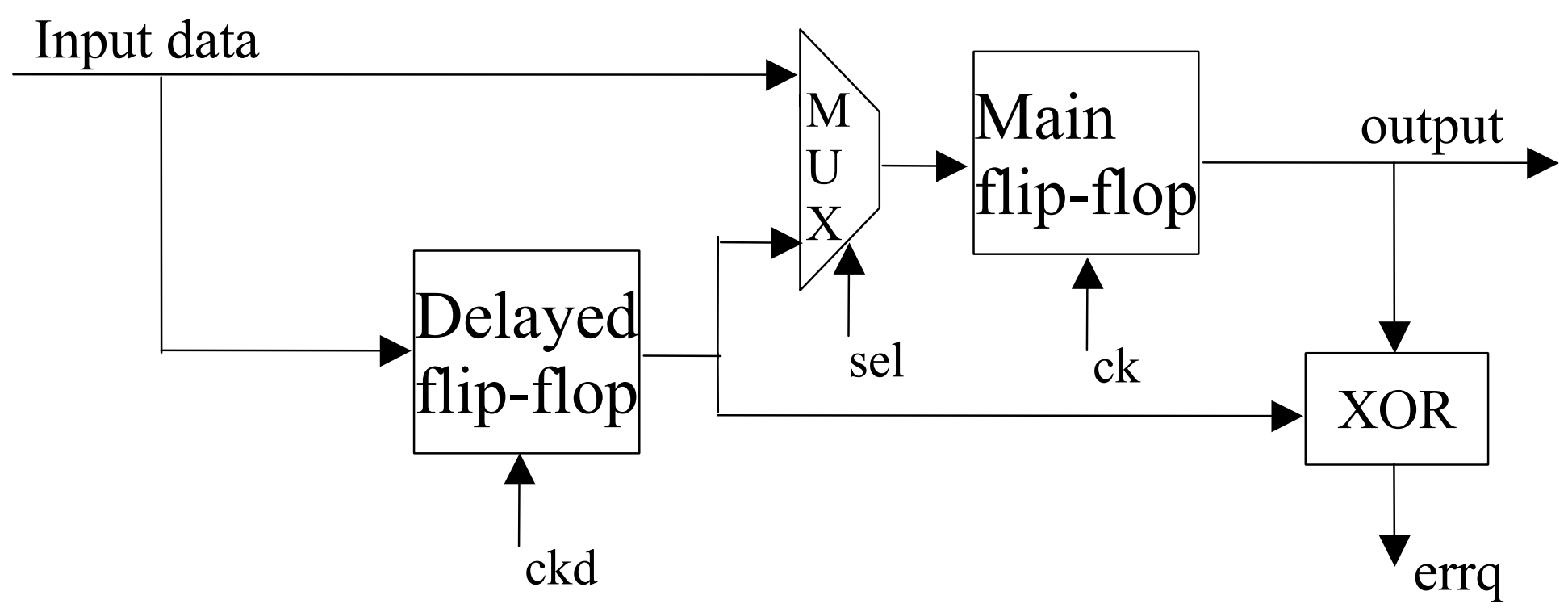

- In normal state, data is captured and sent by main flip-flop

- XOR detects difference in data captured by delayed flip-flop and main flip-flop

- Delay between ck and ckd ensures data bits get sufficient time to reach delayed flip-flop. 


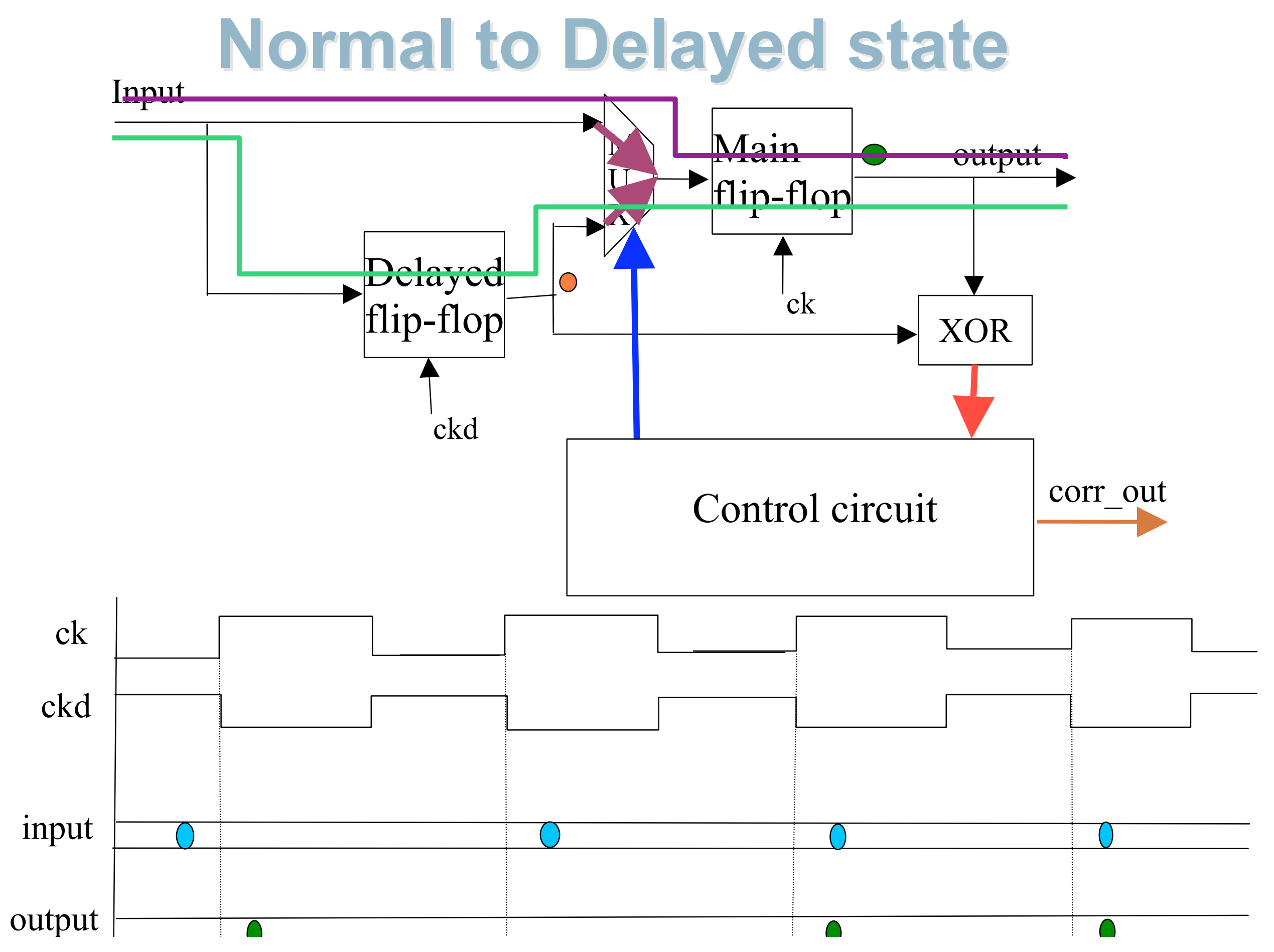




\section{Terror enabled Link design}

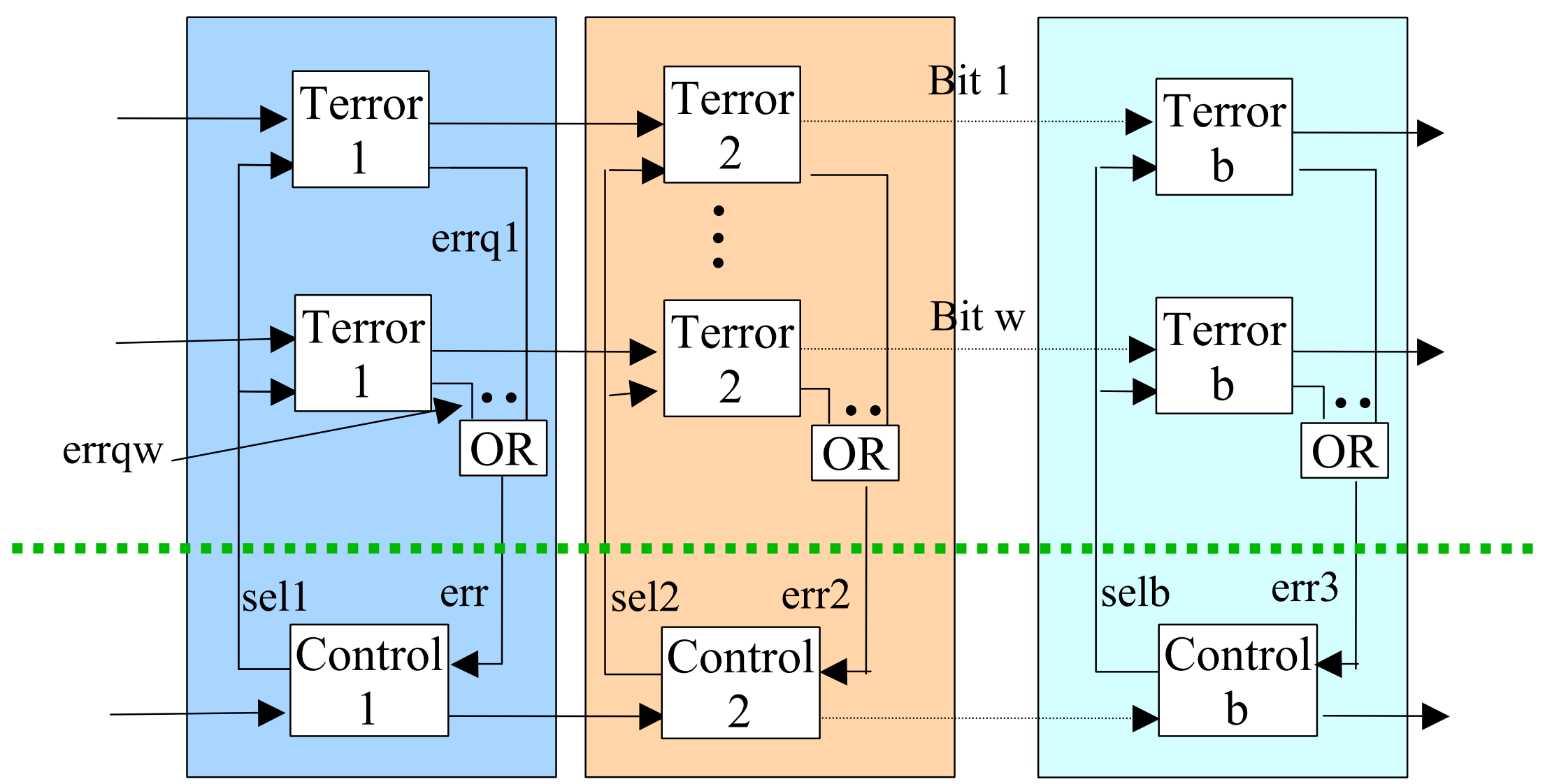

- One error correction circuit common to $w$ buffers

- Decreases overall cost of control circuitry

- errq signals of all buffers (vertically) are Ored and fed to the control circuit.

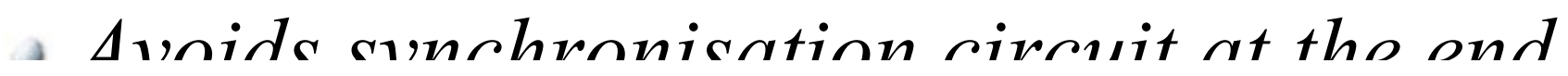




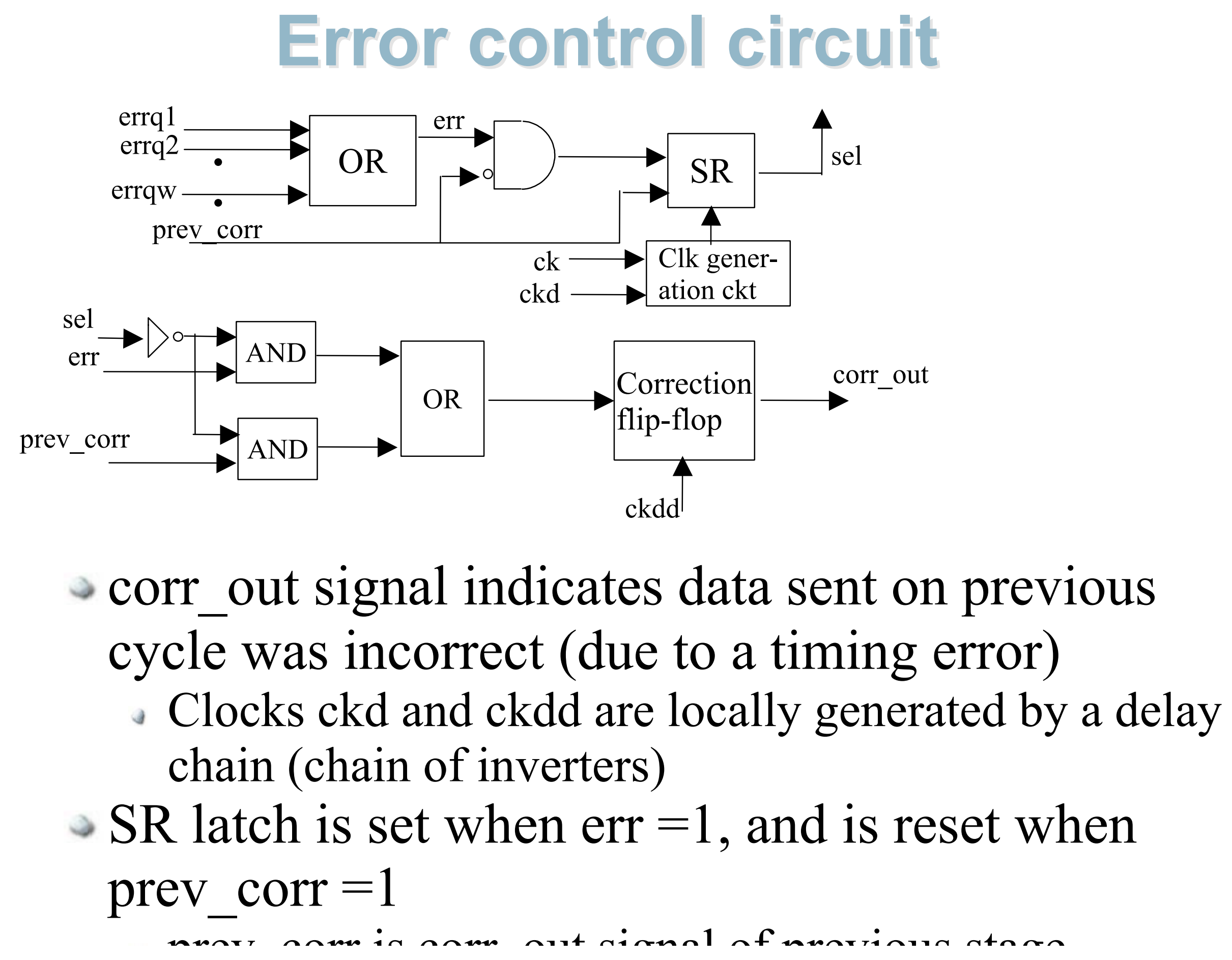




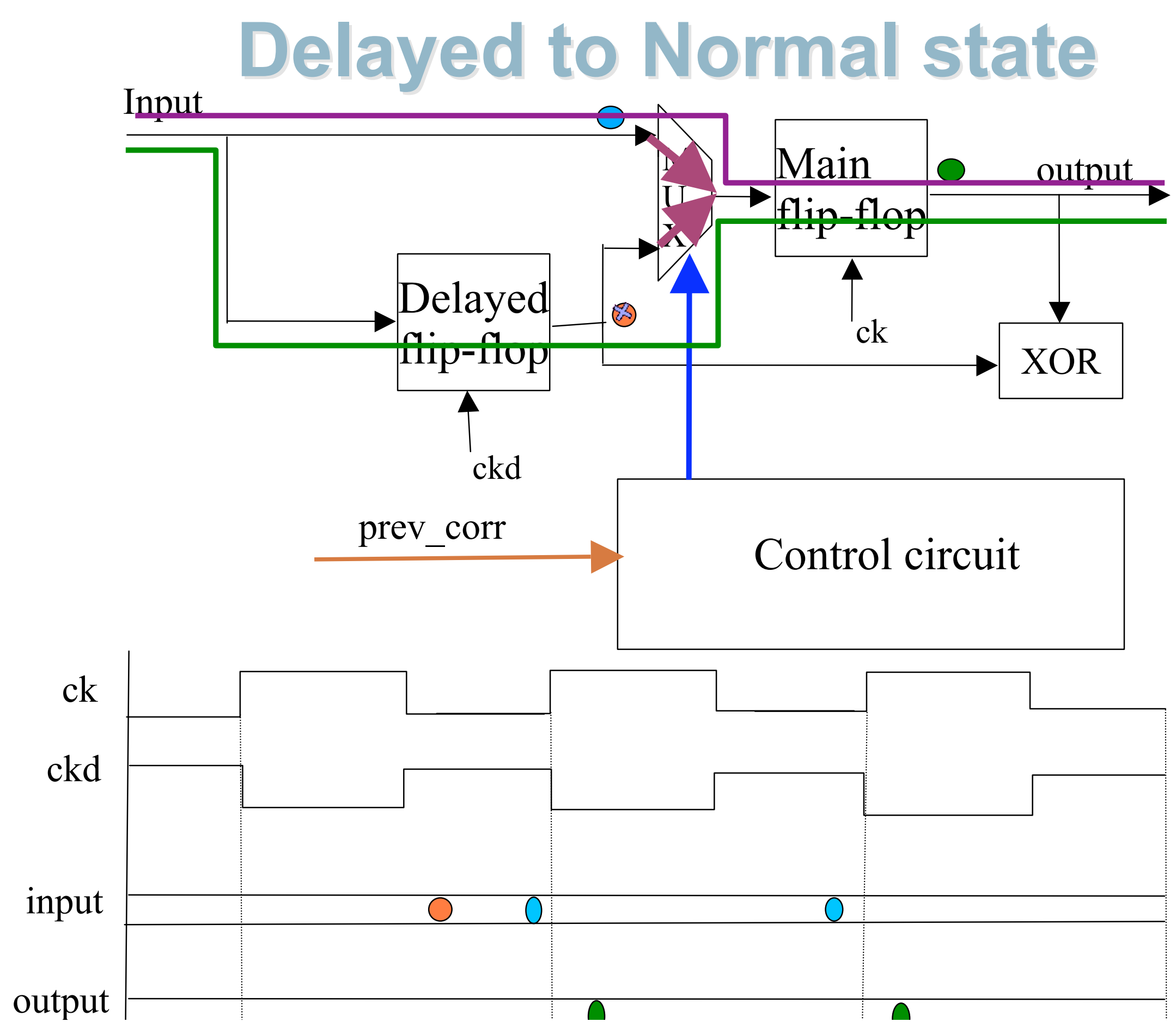




\section{Alternative approaches}

- Teatime [Comp. '04] tracks logic delay and avoids errors by changing the clock frequency.

- Requires complex frequency controller and tracking logic

- Razor [Micro '04] monitors error rate to control power.

- Uses gated clock or flushes pipeline to correct error.

- Favalli et al. [DFT/VLSI '97] use encoded data and decoder at flip-flop input to detect errors.

- Overhead of decoder at each flip-flop input.

- Mousetrap[ICCD '01] uses high speed asynhcronous pipeline. 


\section{Comparison of latency}

- Previous approaches give large latency overheads for high error rates and do not scale well bus widths.

- Error penalty increases linearly with error rate.

- Difficult to correct multiple errors.

- For example, if errors occur each cycle, for $\mathrm{N}$ bits

- Razor [Micro '04] : \% of useful cycles $=\mathrm{N} / 2 \mathrm{~N}=50 \%$

- Terror : $\%$ of useful cycles $=\mathrm{N} /(\mathrm{N}+\mathrm{b})$

where $b=$ no. of buffers on the link

- For $\mathrm{b}<<\mathrm{N}$, the benefits are substantial 


\section{Transistor level design}

- Terror element was designed for 32 bit bus in $100 \mathrm{~nm}$ technology targeted at $1 \mathrm{Ghz}$ frequency.

- Transistor level optimisations were included

- Include 2:1 Mux inside the main flip-flop.

- Use domino OR instead of static OR for errq signals

- Combining AND-OR gates into correction flip-flop

- Using a simple SR latch design.

- Timing overheads were calculated based on SPICE simulations. 


\section{Transistor level design}

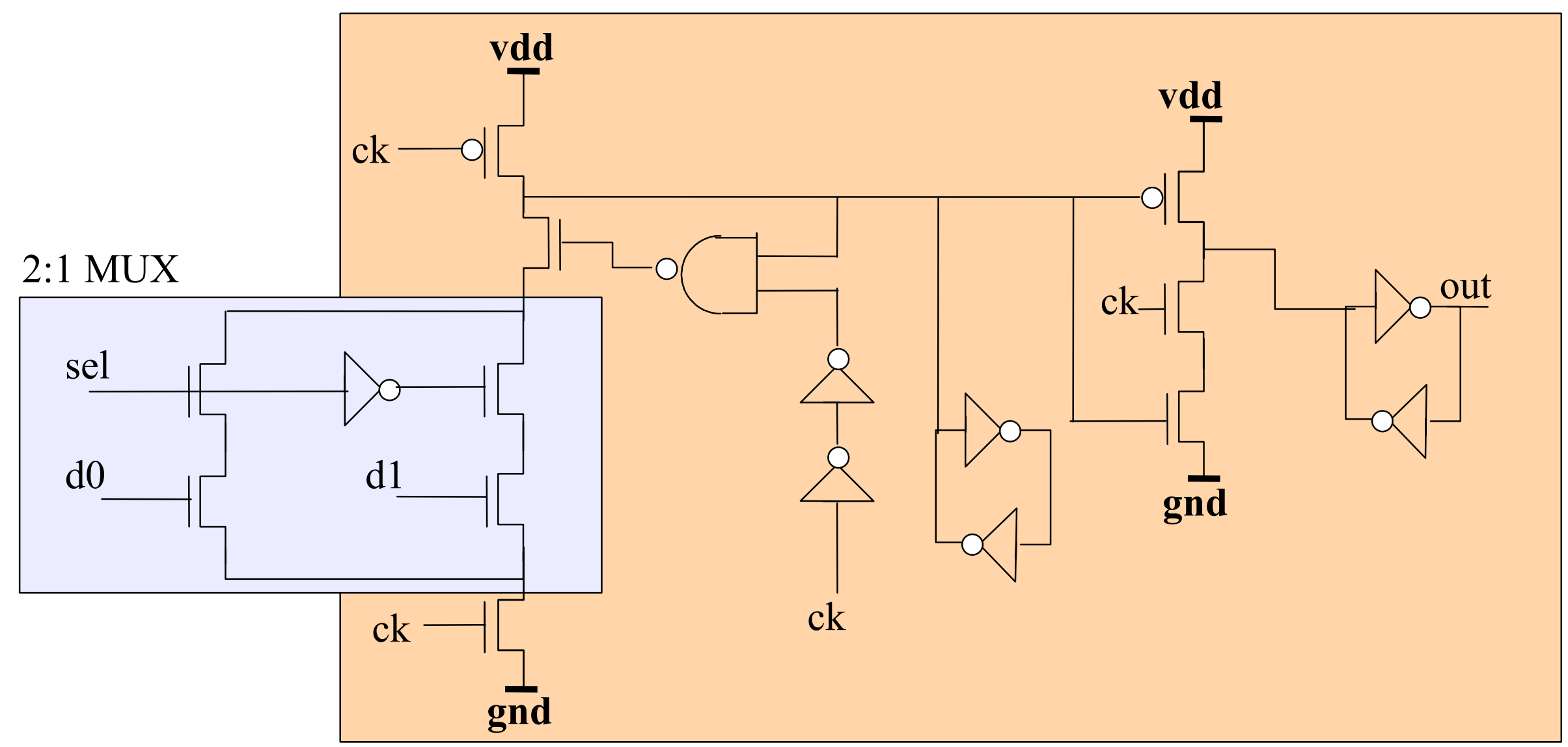

FLIP-FLOP

Schematic showing 2:1 Mux inside a flip-flop 


\section{Timing Overheads}

\begin{tabular}{|l|c|}
\hline Parameter & \% Overhead \\
\hline Hold time & 10.0 \\
\hline Normal- $>$ Delayed & 27.0 \\
\hline Delayed- $>$ Normal & 9.7 \\
\hline
\end{tabular}

- Ideally ckd can be delayed by one cycle

- Practically, ckd delay limited by finite timing delays

- Hold time flip-flop and logic delay involved in going from normal to delayed state and vice-versa.

- Hence range of variation of ckd is

$100-9.7-27-10=53.3 \%$ of cycle

- To simplify, we use 50\% delay variation for ckd

- Clock ckd can be delayed by half clock cycle. 


\section{Simulation results}

- We considered a SoC with on-chip link length of $12 \mathrm{~mm}$ operating at a frequency of $1 \mathrm{Ghz}$

- With conservative design approach assume distance between successive stages as $2 \mathrm{~mm}$.

- Thus number of stages required are 6 .

- A Terror enable pipeline will have 3 stages, ideally (since ckd can be delayed by one cycle)

- Practically ckd can be delayed by 50\%, so number of stages with Terror are 4.

- We plot the receiver latency for different error rates for 1000 data bits in both cases.

- Errors not detected by Terror are corrected by retransmission scheme. 


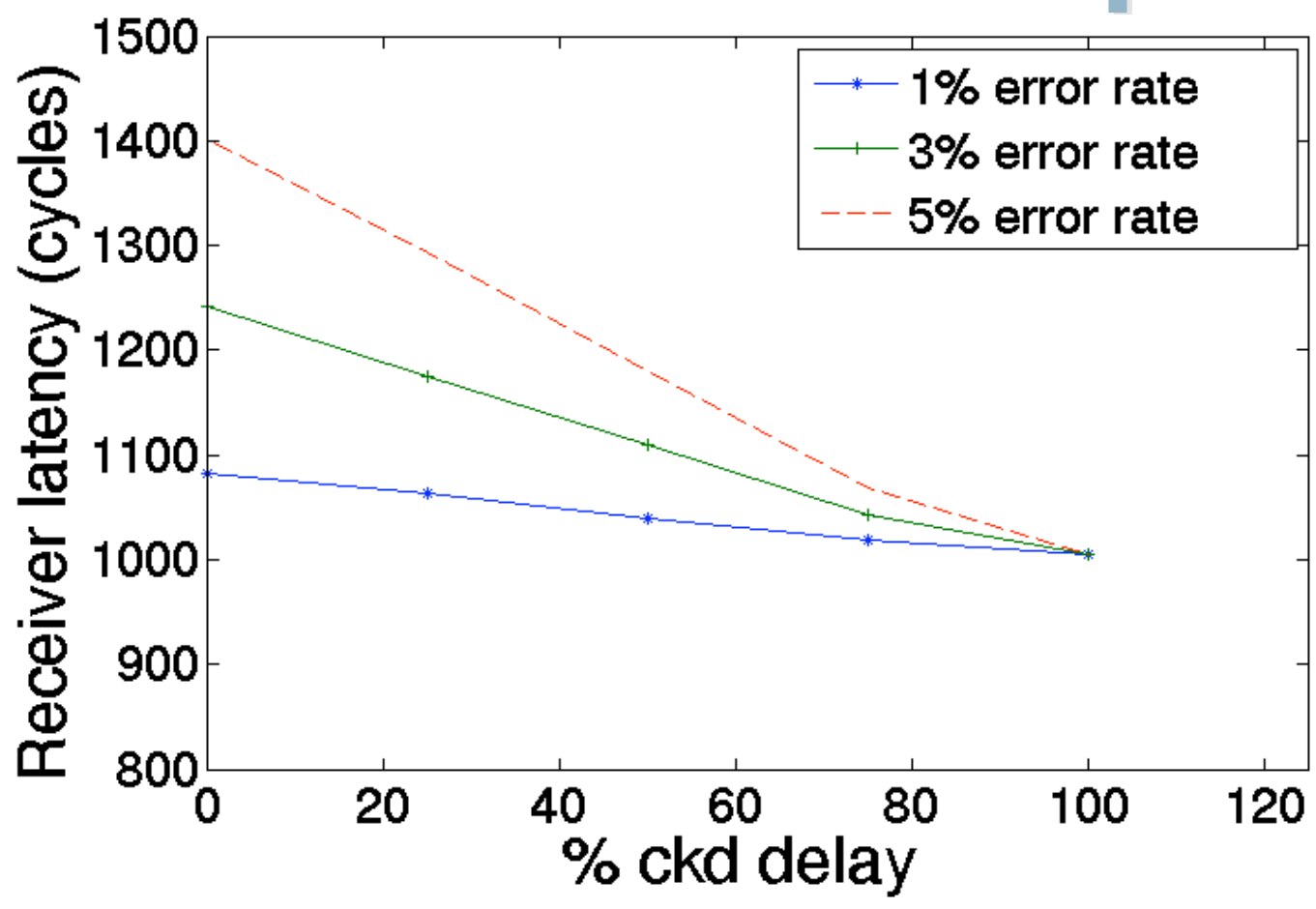

Receiver latency variation with delay between clocks $c k$ and $c k d$ for ideal case

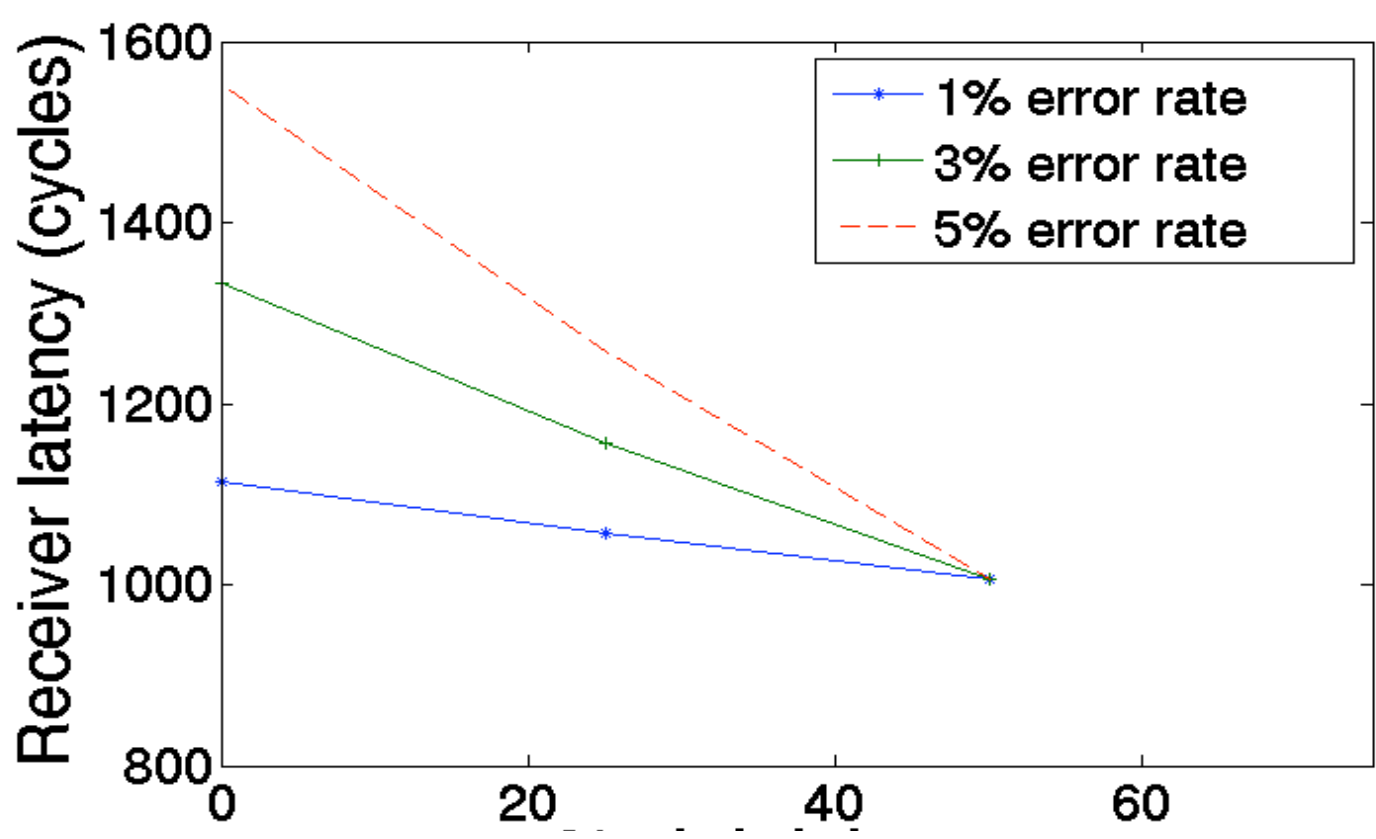

Receiver latency for practical case 


\section{Aggressiveness Vs Latency}

- We define aggressiveness = percentage increase in inter-buffer spacing over conservative design.

- Latency reduces by 33\% for 50\% aggressiveness.

- Plot terminates at 50\% since distance between pipeline stages can only be increased by $50 \%$.

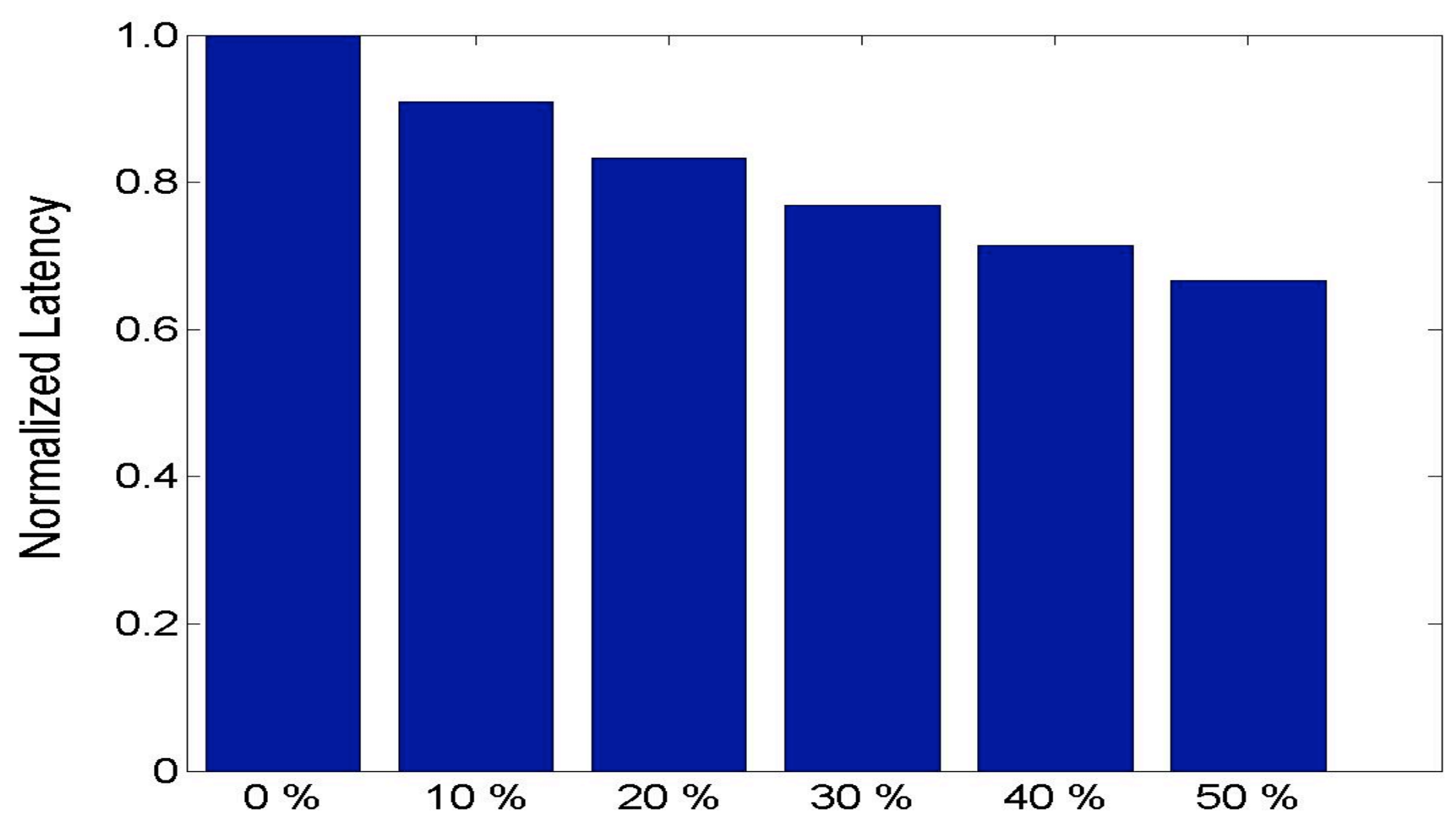




\section{Retransmission Vs Terror Scheme}

- Receiver latency for receiving 1000 bits at $1 \%$ and 5\% error rate is plotted for both schemes.

- Terror scheme gives a 35\% reduction in latency.

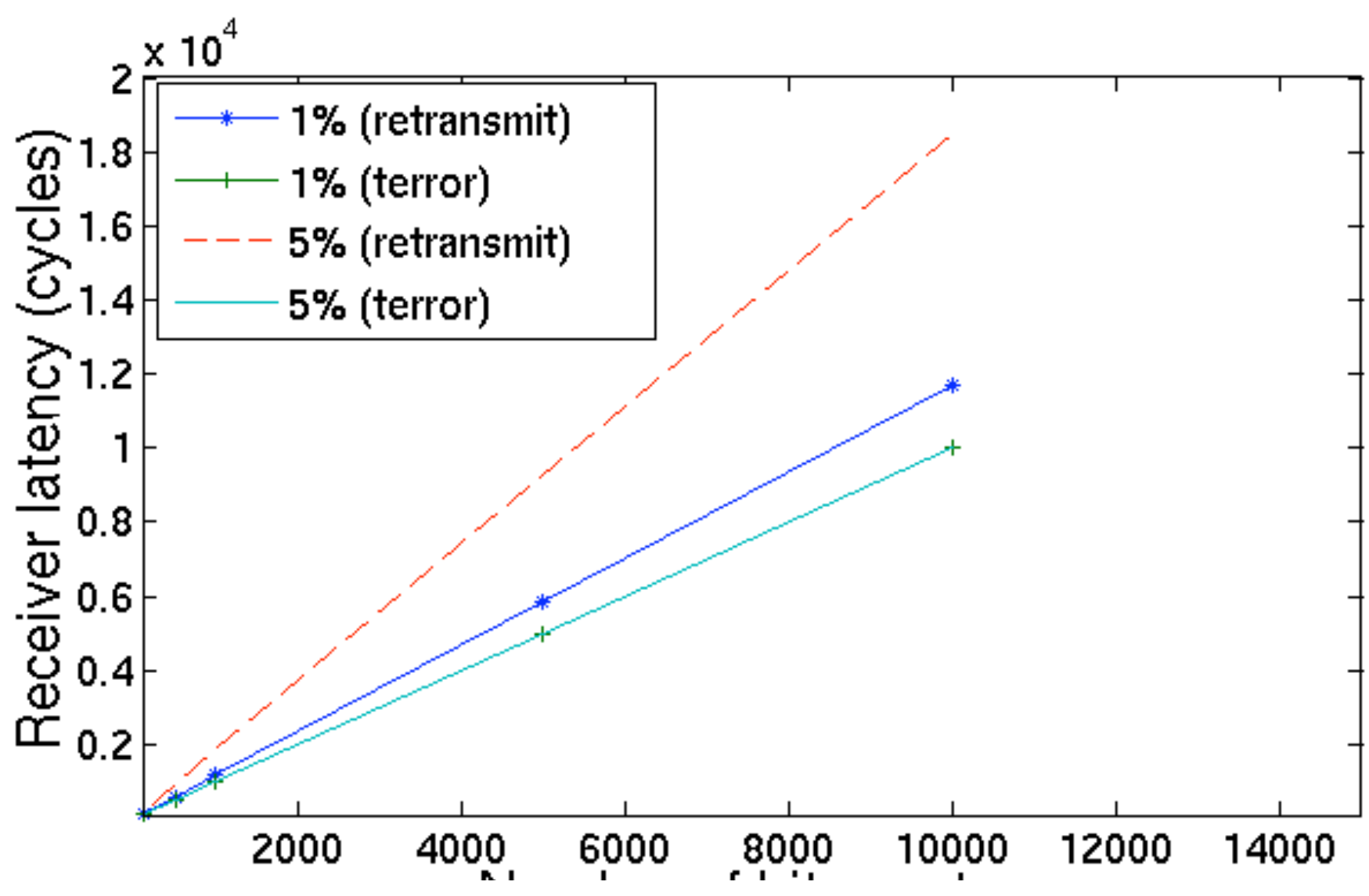




\section{Maximum Penalty Vs Data size}

- Variation in penalty for different error rates and data sizes for a 4-stage pipeline is plotted.

- Maximum penalty is 4 cycles.

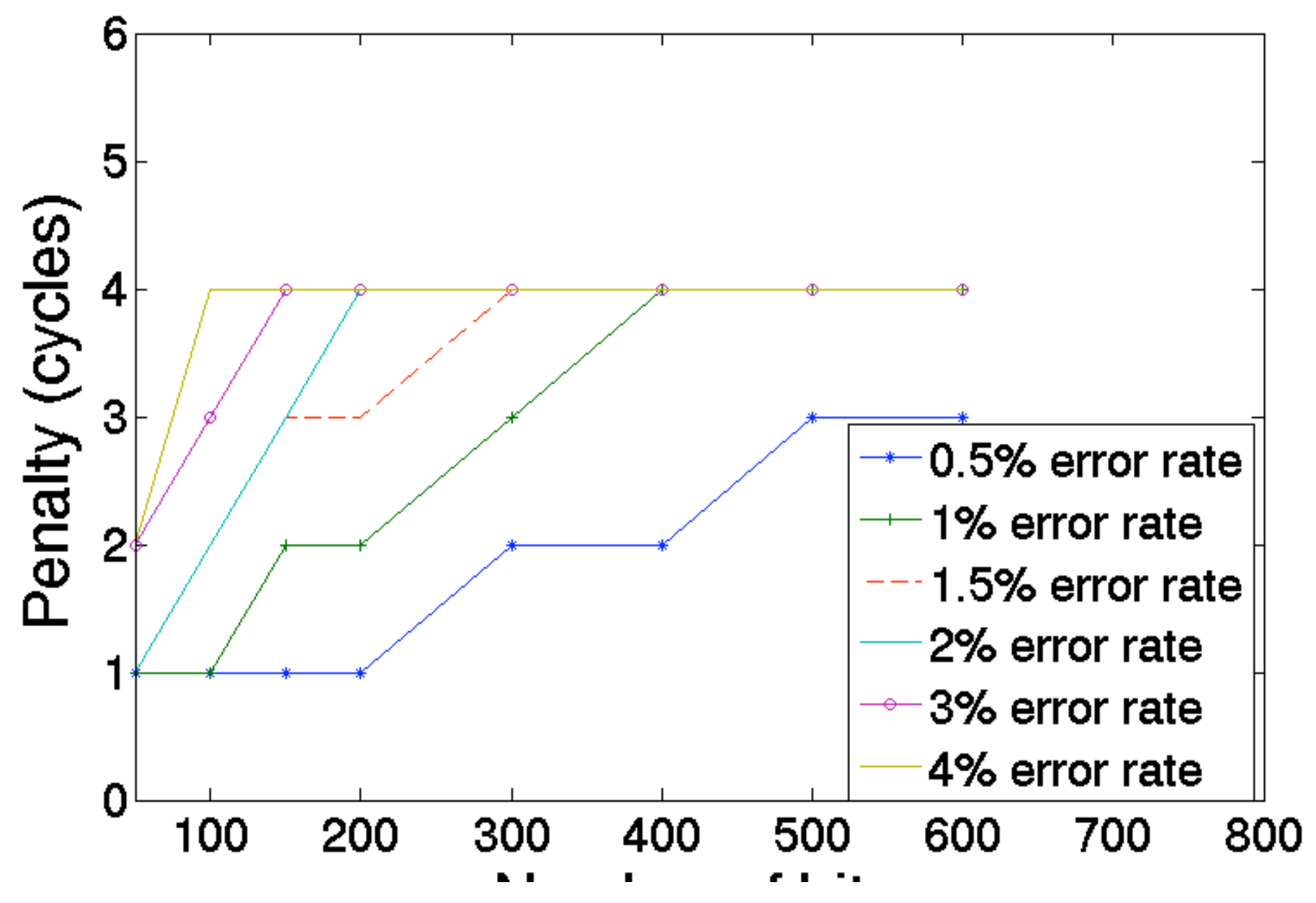




\section{Error Penalty}

$\rightarrow$ Maximum latency is bounded by the number of buffer stages and is not affected by the error rate.

- Latency overhead does not increase for large data sizes or high error rates.

- Typical error correcting schemes degrade at high error rates.

- Terror enabled systems are suitable for high error rate designs.

- This can happen in high noise environment or at low voltage levels.

- Used in aggressive designs, where clock frequency is higher than conservative value or physical spacing between buffers is increased. 


\section{Receiver design}

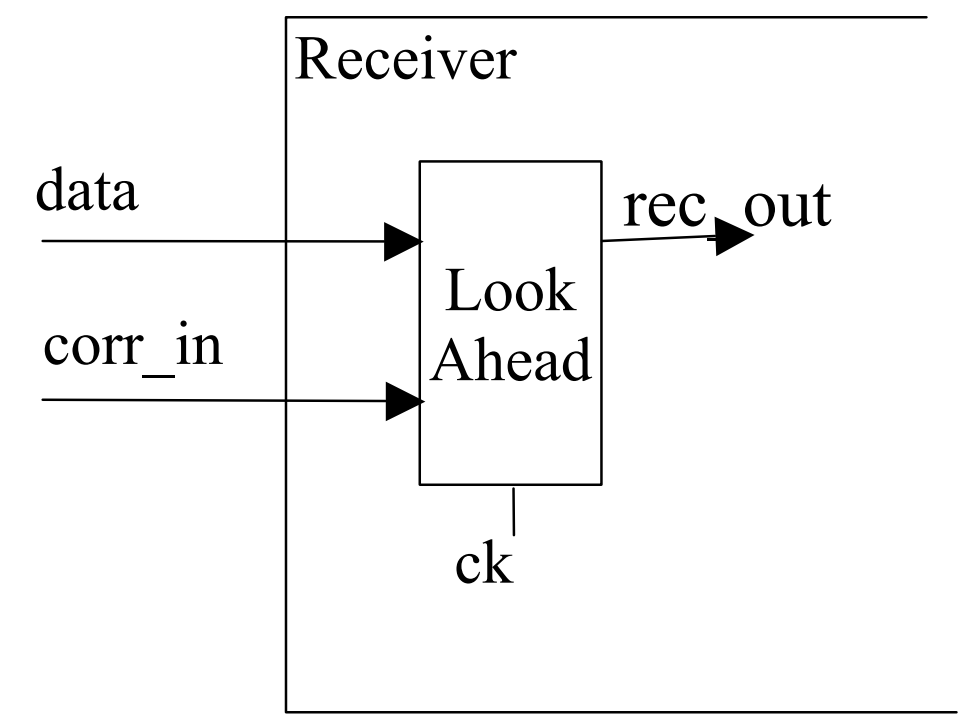

Receiver design

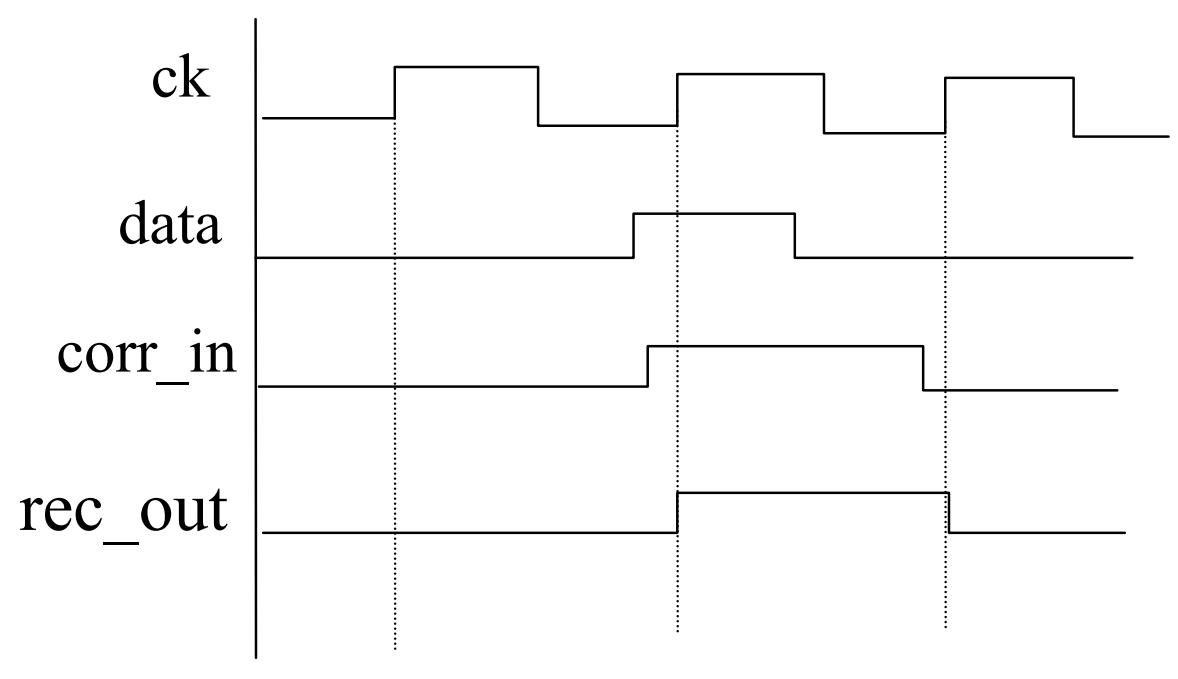

Look-ahead stage operation

- Receiver looks ahead 1 cycle for the data.

- Only first bit incurs 1 cycle penalty since other bits follow in a pipeline fashion.

- 1 cycle penalty can be hidden in switch, it only occurs at the end receiver. 


\section{Area Overhead in typical SoCs}

\begin{tabular}{|l|c|}
\hline Design & Area overhead \\
\hline Merlot & $0.12 \%$ \\
\hline DSP & $0.6 \%$ \\
\hline MIT RAW & $0.86 \%$ \\
\hline Alpha MP & $0.9 \%$ \\
\hline Average & $0.62 \%$ \\
\hline
\end{tabular}

- We estimate area overhead in typical MPSoCs

- Based on increase in gate count due to Terror.

- At a $0.6 \%$ increase in area, we get large latency savings (upto 35\%)

- Power overhead is negligible. 


\section{Conclusion}

- Reliability, delay and throughput of link is affected by unpredictability in link characteristics.

- Increasingly affected by cross-talk, other interferences.

- Terror enabled systems tolerate such variation in link delay and encourage an aggressive design approach.

- Provide a 35\% savings in latency over traditional approach

- Network on Chips (NoCs) a communication centric approach will be required for future SoCs

- Provides scalability and reliability for efficient communication between cores. 


\section{THANK YOU}




\section{EXTRA}




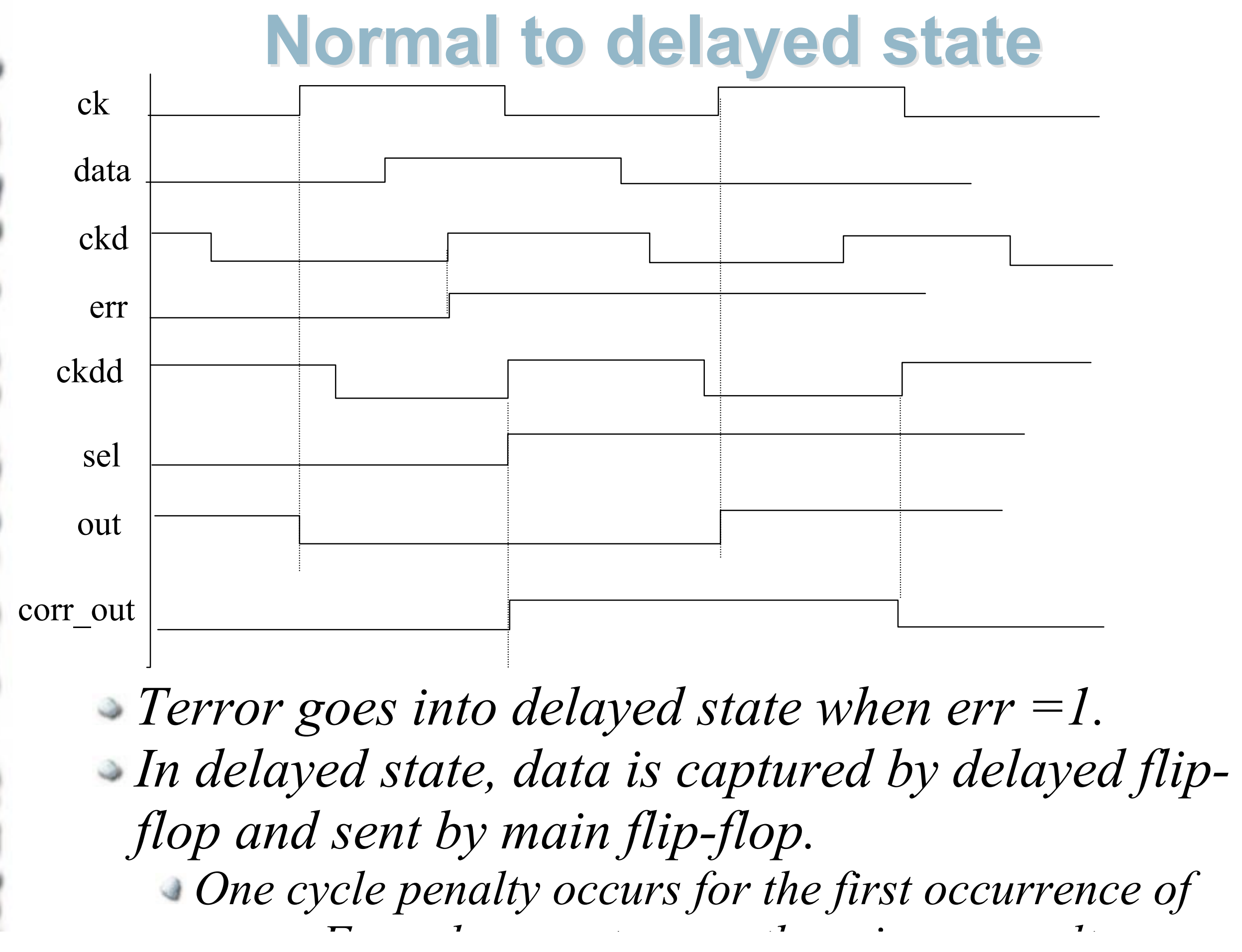




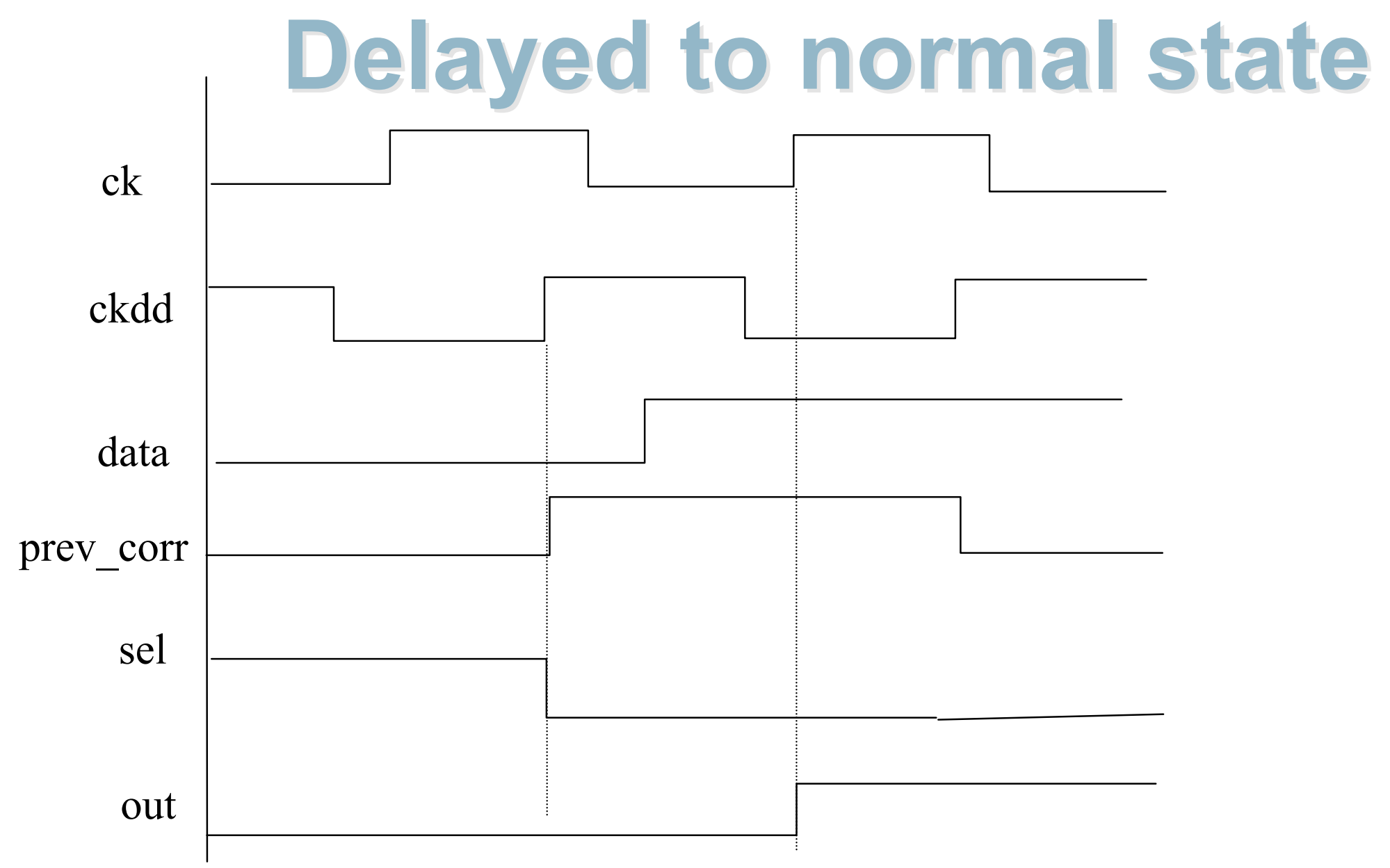

- Terror returns to normal state when prev_corr signal is received.

- Incorrect data captured by delayed flip-flop is not sent.

- For proper operation, prev_corr signal is made

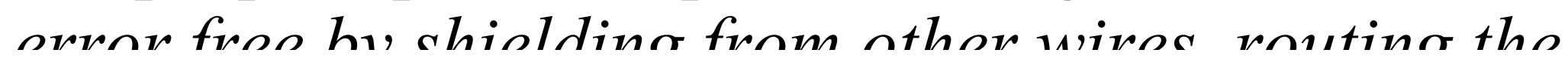




\section{Analysis of Penalty}

- Maximum latency bounded by pipeline stages.

- Average penalty depends upon the when and where timing errors occur along the pipeline.

$$
1 \leq \text { Penalty } \leq \mathrm{b} \text { (for } \mathrm{b} \text { link buffers) }
$$

- One cycle penalty occurs when error in (b-1) $)^{\text {th }}$ stage is absorbed by $b^{\text {th }}$ stage since $b^{\text {th }}$ goes from delayed to normal state

- Worst case (b cycle) penalty occurs when error occurs first in pipeline stage 1 , then in stage 2 , up to $b^{\text {th }}$ stage . 


\section{Timing Analysis}

- Setup time increases due to 2:1 Mux

- $\mathrm{T}_{\text {setup }=} \mathrm{t}_{\text {setup(nominal) }}+\mathrm{t}_{\mathbf{d} \text { (mux) }}$

- Setup time of correction flip-flop

$\mathbf{T}_{\text {setup }}=\mathbf{t}_{\text {and }}+\mathbf{t}_{\text {or }}+\mathbf{t}_{\text {setup(nominal) }}$

- Minimum ckd delay is path delay of err signal

$\mathbf{T}_{\text {ckd }}=\mathbf{t}_{\text {ck-q }}+\mathbf{t}_{\text {xor }}+\mathbf{t}_{\text {domino-or }}+\mathbf{t}_{\text {and }}+\mathbf{t}_{\text {or }}+$ $\mathbf{t}_{\text {setup(nominal) }}$

- Minimum ckdd delay is path delay of sel signal

$\mathbf{T}_{\text {ckdd }}=\mathbf{t}_{\text {SRlatch }}+\mathbf{t}_{\text {mux }}-\mathbf{t}_{\text {setup(nominal) }}$

- Hold time condition of correction flip-flop

$\mathbf{T}_{\text {hold }}<\mathbf{t}_{\text {SRlatch }}+\mathbf{t}_{\text {and }}+\mathbf{t}_{\text {or }}$ 